\title{
Espacio público y espacio político. La ciudad como el lugar para las estrategias de participación"
}

\author{
Julio Alguacil ${ }^{* *}$
}

Resumen: El presente artículo busca, tras una breve mirada del significado histórico de la ciudad, de situarla en el ámbito de la Teoría de las Necesidades Humanas, argumentando cómo la ciudad ha sido el satisfactor sinérgico más importante de las necesidades. Considerando que las necesidades son universales e identificables, se muestra cómo la ciudad es el lugar donde mejor se han satisfecho éstas, y cómo una de ellas, la participación, obtiene un especial significado dado su carácter sinérgico y transversal. No obstante, el desbordamiento de la ciudad con el desarrollo del capitalismo la ha desmembrado, zonificando sus funciones y segregando a los diferentes grupos sociales, perdiéndose el espacio público como espacio político. Reconstruir una democracia urbana, precisa de una (re)vuelta a la ciudad considerando múltiples dimensiones del cambio que son a su vez condiciones y síntomas práxicos para reconstruir el espacio público como espacio político.

Palabras clave: necesidades humanas, derechos de ciudadanía, participación ciudadana, conflicto, segregación, nueva ciudadanía.

\section{Public space and political space. The city as the place for participation strategies}

Abstract: This paper intends, after a brief look on the historic meaning of the city, to situate it in the environment of the Theory of the Human Needs, arguing how the city has been the most important synergic satisfactor of human needs. Considering that needs are universal and identifiable, the city is the best place where these needs have been satisfied, and how one of them, participation, obtains a special meaning given its synergic and transversal character. Nevertheless, the overflow of the city with the development of capitalism has dismembered it, zoning its functions and segregating the different social groups, loosing public space as political space. Reconstructing an urban democracy, needs a (re) return to the city considering multiple dimensions of change which are at the same time conditions and practical symptoms in order to reconstruct the public space as political space.

Key words: human needs, rights of citizenship, civic participation, conflict, segregation, new citizenship.

Recibido: 30.04 .08

Aceptado: 23.05.08

\footnotetext{
* El presente artículo fue encargado y publicado como capítulo de libro por el Instituto Andaluz del Patrimonio Histórico de la Junta de Andalucía en su Colección PH cuadernos: Antonio García García (Coordinador): Espacio público y conjuntos históricos (2008). Consejería de Cultura de la Junta de Andalucía, Sevilla.

**** Universidad Carlos III de Madrid, Getafe, España. Email: julio.alguacil@ gmail.com
} 


\section{La ciudad como satisfactor de las necesidades humanas}

En su sentido histórico y etimológico, la ciudad ha sido el lugar dónde los sujetos han podido encontrarse y asociarse para mejorar sus condiciones de vida de forma común. La gestión compartida en la mejora de las condiciones de vida nos ofrece una primera mirada de la política y así se puede decir con toda propiedad que el origen de la ciudad esta ligado a la política y al propio origen de la democracia, es el espacio del diálogo y, por ello a la vez, del conflicto. En las ciudades se hacen las revoluciones y se producen las innovaciones. En las ciudades se produce el cambio y se construye el conocimiento. La ciudad es, precisamente, el lugar porque es donde se produce la encrucijada del encuentro (la síntesis) entre la diferencia (variedad, heterogeneidad de sujetos, culturas, pensamientos y actividades) y la igualdad (en el acceso a los recursos y en los derechos de ciudadanía), es decir, la ciudad es el lugar de la convivencia que se (re)produce de forma recurrente al combinarse con el conflicto como proceso axiomático que permite avanzar en la satisfacción de las necesidades humanas, aunque, claro esta, siempre en una tensión entre la imperfección de estos supuestos y la conquistas de los mismos.

Originariamente la polis es el lugar construido y apropiado por el sujeto (el hábitat) que alcanza y desarrolla derechos y deberes políticos como estrategia para satisfacer las necesidades humanas, entre ellas la más emblemática en su origen: la de libertad, y que podemos resumir en aquel viejo proverbio aleman de que "el aire de la ciudad nos hace libres", indicando un nuevo modo de vida en sociedad que íba más allá de los estrechos vínculos de parentesco ligados al dominio de la adscripción a la tierra y el vasallaje. Así en un primer estadio de su desarrollo la ciudadanía estaba vinculada a la ciudad. Los ciudadanos lo eran de una ciudad accediendo a los derechos por adquisición de los mismos y no por una transmisión de carácter adscriptivo.

Así la ciudad se descubre como espacio de la política, ya que es donde se produce el encuentro de lo que es diverso, produciéndose a la misma vez el conflicto y el contacto, asociación que promueve el desarrollo de los complejos procesos sociales para superarlo, para construir nuevas síntesis conflictivas-convivenciales, y eso es así porque irremediablemente se produce la interdependencia de los elementos que hacen la ciudad. La participación de las partes, de los elementos, de los actores, es lo que permite incorporarse al juego de la política en un sentido de creación permanente y en una orientación que hace de la satisfacción de las necesidades una estrategia humana relacional.

La mirada relacional se produce en la ciudad. "La ciudad -según Barry Clarke- no era para los griegos una reunión de individuos previamente autónomos sino un conjunto de personas que se concebían a sí mismos en función de su pertenencia a la ciudad. No eran individuos externamente relacionados con la ciudad sino personas internamente relacionadas unas con otras y con la ciudad" (Barry, 1999: 142). La autonomía personal 
que confiere la ciudad se construye, paradójicamente, desde la dependencia que el sujeto tiene del medio social $\mathrm{y}$, particularmente, de la propia interactividad de las relaciones urbanas, es decir, de la interdependencia. Precisamente la conciencia del yo individual que se adquiere en la ciudad se produce a través de la alteridad u otredad, nuestra conciencia de sí "se nutre de lo que le altera" (Delgado, 1999: 15). Dicho de otro modo, las necesidades humanas se satisfacen en la ciudad merced a la interactividad que en ella se ocasiona entre sus heterogéneos componentes, y esto nos ayuda también a entender como las necesidades conforman un sistema complejo de tal suerte que la satisfacción de cada una de ellas depende de la satisfacción adecuada de las demás.

Queremos adoptar este punto de partida que nos permite pensar que la participación es un derecho porque es una necesidad humana. Nos situamos desde el enfoque de la teoría de las necesidades humanas (Doyal, Gough, 1994) y del desarrollo a escala humana (Max-Neef, Elizalde, Hopenhayn, 1986). Ambos, enfoques son bastantes equivalentes y contrastan abiertamente con teorías muy asentadas como la perspectiva jerárquica de las necesidades de Maslow (1975). Vienen a plantear que las necesidades humanas son pocas, finitas, identificables, clasificables, sinérgicas (conforman un sistema) y universales, es decir son iguales para cualquier ser humano independientemente de la época histórica que le haya correspondido vivir o de la cultura donde se haya socializado. Lo que varía de una época a otra y de una cultura a otra, son los procedimientos e instrumentos a través de los cuales se satisfacen esas necesidades, es decir, lo que estos autores denominan satisfactores.

Los satisfactores pueden ser de muy distinta naturaleza: desde satisfactores destructores o violadores (que al ser aplicados con la intención de satisfacer una determinada necesidad, terminan afectando negativamente en la satisfacción de esa u otras necesidades para nosotros mismos o para otros sujetos) hasta satisfactores sinérgicos (donde el procedimiento por el que se satisface una determinada necesidad estimula y contribuye a la satisfacción de otras necesidades para uno mismo y para otros sujetos en el presente y en el futuro). Es decir, la satisfacción de una necesidad humana no puede basarse en acciones que impliquen la no-satisfacción de esa misma necesidad, o de otras necesidades, en el futuro o para otros seres humanos ubicados en otros lugares o socializados en otras culturas. Por el contrario, cualquier satisfactor de una necesidad determinada debe procurar el favorecimiento de la satisfacción de otras necesidades de orden ontológico distinto, o en todo caso, la forma de satisfacer una necesidad nunca debe ir en menoscabo de la satisfacción de otras necesidades o de la satisfacción de la misma necesidad para otros sujetos.

Este último razonamiento plantea una reciprocidad simétrica entre las necesidades que conforman un sistema. Para Antonio Elizalde y Manfred Max-Neef el sistema de necesidades combina categorías axiológicas (nueve necesidades: subsistencia, protección, afecto, comprensión, participación, creación, recreo, identidad y libertad) con categorías existenciales (ser, tener, hacer, relaciones). Todas ellas las podríamos considerar como derechos humanos, y cada una de ellas, sí es satisfecha a través de satisfactores sinérgicos, contribuye transversalmente a la adecuada 
satisfacción de las demás. Quizá la más relevante en este sentido es la necesidad de participación ya que ésta interviene directa y transversalmente, optimizando el acceso a la satisfacción de las demás necesidades, es la más radical. De hecho Len Doyal e Ian Gough la identifican como autonomía crítica (derecho a comunicar, a proponer, a disentir, a decidir, a compartir), en definitiva, capacidad para participar. De este modo, las necesidades de subsistencia, de protección, de afecto, de entendimiento, de creatividad, de recreo, de identidad y de libertad no podrían optimizarse sin la participación de los sujetos en la gestión de la ciudad y ésta es entonces, posiblemente, el satisfactor más complejo y efectivo de las necesidades humanas, porque la ciudad produce relación, comunicación, conocimiento, pensamiento e innovación.

\section{MATRIZ DE NECESIDADES APLICADAS AL SATISFACTOR CIUDAD $\rightarrow$ ESPACIO PÚBLICO}

\begin{tabular}{|c|c|c|c|c|}
\hline & \multicolumn{4}{|c|}{ NECESIDADES SEGÚN CATEGORÍAS EXISTENCIALES } \\
\hline $\begin{array}{l}\text { NECESIDADES SEGÚN } \\
\text { CATEGORÍAS } \\
\text { AXIOLÓGICAS }\end{array}$ & $\begin{array}{l}\text { SER (SUJETO DE } \\
\text { DERECHOS) }\end{array}$ & TENER & HACER & RELACIONES \\
\hline SUBSISTENCIA & $\begin{array}{l}\text { Derecho a la vida, derechos } \\
\text { ambientales, Derecho a la salud }\end{array}$ & $\begin{array}{l}\text { Alojamiento, trabajo, salud, } \\
\text { alimentos }\end{array}$ & $\begin{array}{l}\text { Descansar, buena } \\
\text { alimentación }\end{array}$ & $\begin{array}{l}\text { Entorno vital, medio } \\
\text { social }\end{array}$ \\
\hline PROTECCIÓN & Derecho sociales y económicos & Seguridad. Solidaridad & Cooperación & $\begin{array}{l}\text { Entorno social } \\
\text { integrador }\end{array}$ \\
\hline AFECTO & Derechos relacionales. & $\begin{array}{l}\text { Relaciones familiares, redes } \\
\text { sociales, generosidad }\end{array}$ & $\begin{array}{l}\text { Expresar } \\
\text { emociones, } \\
\text { compartir }\end{array}$ & $\begin{array}{l}\text { Vida privada, } \\
\text { intimidad, amistad, } \\
\text { vecindad, familiar. }\end{array}$ \\
\hline ENTENDIMIENTO & Derechos políticos y sociales & $\begin{array}{l}\text { Acceso a la información. } \\
\text { Soportes de comunicación. } \\
\text { Espacio urbano legible y } \\
\text { accesible }\end{array}$ & $\begin{array}{l}\text { Reflexionar, } \\
\text { dialogar, } \\
\text { comunicar. }\end{array}$ & $\begin{array}{l}\text { De confianza, de } \\
\text { compromiso }\end{array}$ \\
\hline PARTICIPACIÓN & \begin{tabular}{lccc} 
Derechos & de & \multicolumn{2}{c}{ ciudadanía } \\
Derecho & al & "buen" \\
gobierno, Derecho a la & a & & \\
producción del espacio & &
\end{tabular} & $\begin{array}{l}\text { Responsabilidad, } \\
\text { Capacidad de decisión. } \\
\text { Capacidad de gestión }\end{array}$ & $\begin{array}{l}\text { Compartir, } \\
\text { decidir, asociarse }\end{array}$ & $\begin{array}{l}\text { De reciprocidad, } \\
\text { consenso, negociación, } \\
\text { co-responsabilidad }\end{array}$ \\
\hline CREACIÓN & $\begin{array}{l}\text { Derecho al trabajo y a la } \\
\text { formación }\end{array}$ & $\begin{array}{l}\text { Formación, } \quad \text { Información. } \\
\text { Soportes, } \\
\text { equipamientos polivalentes }\end{array}$ & $\begin{array}{l}\text { Estudiar, } \\
\text { Analizar Diseñar, } \\
\text { Planificar Innovar }\end{array}$ & $\begin{array}{l}\text { De intercambio, de } \\
\text { colaboración, } \\
\text { cooperación }\end{array}$ \\
\hline RECREO & $\begin{array}{l}\text { Derecho al tiempo libre. } \\
\text { Derecho a la libre circulación y } \\
\text { al espacio público }\end{array}$ & $\begin{array}{l}\text { Espacios libres, tiempo } \\
\text { liberado, equipamientos. }\end{array}$ & Imaginar, crear & $\begin{array}{l}\text { De proximidad, } \\
\text { amistad, vecindad, } \\
\text { familia }\end{array}$ \\
\hline IDENTIDAD & Derechos culturales & $\begin{array}{l}\text { Autoestima. Ámbitos de } \\
\text { pertenencia, } \quad \text { espacios } \\
\text { simbólicos, apropiados }\end{array}$ & $\begin{array}{l}\text { Apropiarse, } \\
\text { participar, } \\
\text { afirmarse, } \\
\text { tolerarse }\end{array}$ & $\begin{array}{l}\text { Múltiples con los otros. } \\
\text { Interculturalidad }\end{array}$ \\
\hline LIBERTAD & Derechos políticos y jurídicos & $\begin{array}{l}\text { Autonomía. Capacidad de } \\
\text { elección }\end{array}$ & Convivencia & $\begin{array}{l}\text { Democráticas, } \\
\text { tolerantes, abiertas }\end{array}$ \\
\hline
\end{tabular}

Elaboración adaptada sobre la matriz de necesidades de Max-Neef, Elizalde y Hopenhayn.

"Desarrollo a escala humana" (1986: 42) 
El redescubrimiento y la identificación de las necesidades por parte de los propios sujetos implicados en los procesos sociales asienta un significado axiomático en la perspectiva humanista de las necesidades. Desde este punto de vista la participación de los sujetos en la satisfacción de las necesidades es entendida como la capacidad para decidir sobre los asuntos que les afectan directamente, y es en sí una necesidad humana básica que orienta los derechos de ciudadanía, de acceso al conocimiento y de acceso a la comunicación. El conocimiento y la comunicación dan paso a la conciencia completando las tres ces que permiten la acción social en un sentido participativo y de corresponsabilidad. De este modo la participación se nos antoja como la más transversal de las necesidades humanas y con mayor capacidad sinérgica. Los satisfactores de la participación son muy diversos (la ciudad y el espacio público, como espacio político, pueden considerarse como tales), pero todos ellos precisan de una naturaleza de base sinérgica para estimular la satisfacción de las otras necesidades

Así, por ejemplo, la necesidad de "protección" no será satisfecha plenamente sin la concurrencia de otra necesidad humana como es la participación o la autonomía crítica de los sujetos, de tal modo que cualquier necesidad no se podrá optimizar sin la adecuada satisfacción de otras necesidades. La falta de participación limita el acceso a la comunicación, al conocimiento, a la conciencia, a los espacios públicos y esto restringe la seguridad personal. Nos interesa particularmente poner énfasis en la idea de que ninguna necesidad se podrá satisfacer de forma óptima sin la participación de los sujetos implicados en los procesos donde se inscriben.

La participación para ser genuina, para ser una necesidad que se satisface adecuadamente debe tener, ser, hacer y relacionar en un proceso (que no en un momento) recurrente e inagotable capaz de: transformar (cambiar para mejorar las condiciones de existencia), reflexionar (pensando en los efectos e impactos a largo plazo), implicar (al mayor número de colectivos y sujetos, especialmente a los más desfavorecidos), articular (poner en relación reciproca los distintos actores, colectivos y territorios), construir (crear conjuntamente), conocer (la realidad, los recursos y sus potencialidades), aprender (educar en el diálogo, el consenso y la solidaridad), comunicar y comunicarse (con los iguales y con los diferentes), habilitar (cualquier sujeto puede acceder a las habilidades políticas), gratificar (generando sentimiento de satisfacción y de utilidad) y exigir (los procesos de participación como derecho).

Como el resto de las necesidades, la participación, se satisface en primer lugar en el ámbito de la vida cotidiana, en el ámbito urbano, donde las estructuras gubernativas y societarias deben interpretarse como satisfactores sinérgicos con capacidad para procurar, por su proximidad e interactividad, nuevas oportunidades políticas para una participación genuina, integral e inclusiva, siendo susceptible de crear una estructura común de acción política (Held, 1997). Es decir, relacional y dialogal. Precisamente, la democracia participativa, que es autonomía crítica, se basa en la participación como un conjunto de procedimientos y procesos relacionales donde los agentes entran en relación 
simétrica y reciproca, de comunicación, de cooperación y de corresponsabilidad. El acoplamiento de los agentes que intervienen en la vida social a esta perspectiva de la participación, como necesidad y como derecho, es lo que permite recuperar el sentido transversal y relacional de la participación dotándole de su sentido innovador y transformador.

La participación, en definitiva, es lo que permite acceder al estatus de ciudadanía. Siguiendo a Jordi Borja "ser ciudadano es sentirse integrado física y simbólicamente en la ciudad como ente material y como sistema relacional, no sólo en lo funcional y en lo económico, no sólo legalmente. Se es ciudadano si los otros te ven y te reconocen como ciudadano" (Botja, 2003: 28), de tal modo que no hay plena participación, no hay plena democracia urbana, si algún colectivo o grupo de sujetos identificado por atributos comunes adscritos (etnia, religión, origen nacional, edad, género etc.) queda excluido del estatus de ciudadanía.

La participación, en consecuencia, es el nexo que asocia lo público (diversidad de actores) y lo político (estrategia de puesta en común entre los actores), y ello tiene su plasmación en el territorio, ya que la organización del mismo y la ordenación de las relaciones que soporta son inherentemente políticas. Pero, además, el espacio público y el espacio político se solapan, se confunden, se fusionan. Precisamente el nexo de unión entre uno y otro conforma la polis, la ciudad originaria. La ciudad es entonces una síntesis construida por la fusión entre la forma física y la cultura, entre el entorno y el medio social, retroalimentándose y, en consecuencia, modificándose mutuamente de manera permanente: lo conductual determina el espacio físico, el espacio público; y la forma del espacio público determina las conductas y las relaciones sociales. De aquí que la praxis urbana, entendida como la síntesis resultante de la combinación de la acción política (participativa) y el espacio urbano, la podamos considerar como la actividad mediante la cual el sujeto transformador se transforma al participar en la transformación del espacio urbano (Alaguacil, Montanes, 1998 : 139). Esto se concreta en los espacios públicos como lugares donde cada uno siente personalmente que los otros pueden, deben, y se apropian del espacio igual que mi persona estableciendo complicidades y relaciones densas. En contraposición, en los "No-lugares" (Auge, 1993), impersonales, no apropiables, no es posible la alteración pues son espacios de mero tránsito, donde difícilmente se pueden generar sentimientos de pertenencia y relaciones con los otros sujetos que también son transeúntes.

\section{La ciudad ese complejo espacio público y (por ello) conflictivo, es decir, político}

El espacio público es lugar donde todo ciudadano tiene derecho a circular, a estar y hacer, en contraste con el espacio privado donde el paso, la estancia y la cre-acción están restringidas. El poder transitar remite a la libertad de movimiento, el poder estar remite a la apropiación del espacio y el poder hacer remite a la participación en el 
espacio público. Enfatizando este último aspecto nos lleva a la consideración de que el espacio público es siempre un espacio colectivo donde se encuentran los diferentes, los actores diversos, las partes que comparten el espacio y que al hacerlo lo elevan a la categoría de público y colectivo, es decir, los diferentes toman conciencia de la diferencia porque son susceptibles de encontrarse, interactuar y finalmente interaccionar, por ello el compartir el espacio creativamente significa estar en un lugar, ser parte, sentirse parte, tomar o tener parte y hacer lugar. En consecuencia, el espacio público, es aquel espacio de propiedad pública, y de dominio y uso público. La propiedad pública infiere un sentido político, el dominio público un sentido cultural y el uso público un sentido social.

La participación es lo que permite la apropiación del espacio público de manera compartida, el sentimiento de ser mío, nuestro, sin excluir de ese sentimiento a los otros ciudadanos, del sentimiento que el espacio urbano también es tuyo o suyo, es lo que hace del espacio público un espacio colectivo. Esta idea nos ayuda a adoptar una definición radical del espacio público de acuerdo con Hernández Aja: "el espacio público, desde la condición de ciudadanía, será aquel en el que se expresan las distintas visiones de la ciudad, en el que se construyen los acuerdos y limitaciones entre los distintos grupos sociales e intereses, en permanente construcción pero en el que siempre se garantiza la identidad del distinto como garantía de la identidad propia... en el que nos podemos encontrar con el resto de los ciudadanos, en el que nadie sobra ni debe ser rechazado" (Hernández AJA, 2003: 18).

Ello no quiere decir que el espacio público no este regulado. Esta regulación se hace por el Estado, por la administración local (por ordenanzas municipales, por ejemplo), pero más particularmente ¿por quién está regulado el espacio público? La propiedad pública es colectiva, pero se gestiona y se controla, en el espacio urbano, a través del gobierno local en el que se reconocen los ciudadanos más cuanto más proximidad y participación se produce en este nivel. Si los políticos locales y las políticas locales son capaces de establecer estrategias de democracia participativa encaminadas a habilitar a los ciudadanos para que participen en la decisión de las políticas públicas urbanas (y en la gestión del espacio urbano) estaremos frente un primer nivel de participación ciudadana. Si la composición y disposición de los espacios y una regulación participada de los mismos permite un dominio público de naturaleza inclusiva e intercultural podremos hablar de un verdadero domino público que permite la apropiación del espacio por parte de los ciudadanos diferentes entre sí y, finalmente, si el uso del espacio público permite la expresión política y cultural de una ciudadanía que solo podemos ya considerar como plural, podemos considerar al espacio público como el lugar donde se desarrolla un interactividad que permite la implicación e integración de todos los actores en la aplicación de los derechos de ciudadanía. El espacio público es más público y más colectivo si motiva la participación y menos si inhibe de la misma.

Así, un espacio público plenamente colectivo, al servicio de la ciudadanía democrática, genera sentimiento de pertenencia al hacer de éste un símbolo de la 
ciudadanía, y acoge según momentos y características del mismo, la manifestación cultural, la manifestación política, la fiesta y el juego, la música y el teatro, el arte y el intercambio de la economía popular (el mercadillo, el rastrillo, el músico y el actor callejero...), el uso de diferentes colectivos (culturales, de género, de edad), espontáneos o planificados, en definitiva, el uso pleno del espacio público no puede ser sin un fuerte carácter polivalente y una complejidad accesible, donde el uso puede ser más o menos planificado, o más o menos espontáneo, según sea apropiado por redes sociales o por grupos más organizados como las asociaciones. Siguiendo a Hernández Aja "los proyectos sociales de base democrática se han reflejado sobre la ciudad mediante la creación de espacios colectivos" (Hernández Aja, 2000: 81), por ello la producción social del espacio, la creación del espacio urbano es una conquista permanente que se produce a través de las sucesivas revoluciones democráticas (BORJA, 2003: 33).

Por otro lado, también, la ciudad es el espacio del conflicto, la ciudad deja riendas sueltas a las paradojas, donde se produce el encuentro de las diferencias y de las divergencias, de los intereses contrapuestos, que se tocan, que se mezclan, que confrontan, que se complementan, y así se modifican mutuamente. El perímetro de la ciudad es el espacio de la cohabitación de diferentes grupos, clases, culturas, redes, y sus espacios internos, el ágora, el mercado, la calle, son los espacios públicos, los espacios que se comparten, donde se encuentran, que se viven colectivamente, es decir, los espacios donde se produce el intercambio, y en consecuencia se estimula la convivencia y también donde se produce de manera inevitable el conflicto.

El conflicto, entonces, en la ciudad no se puede ni se debe esconder, precisamente es en la ciudad donde el conflicto se hace evidente y se puede reconocer, única manera, por otro lado, de sentar las bases para superarlo, aunque, dejando puertas abiertas a nuevas dialécticas y a nuevas dialógicas, a nuevas contradicciones y a nuevos diálogos. Precisamente "la gran aportación de la política democrática es que no escamotea el conflicto, sino que lo canaliza para evitar la arbitrariedad; no pretende erradicar el poder (lo que sería sospechoso), sino proporcionar espacio adecuados para un ejercicio efectivo de la discusión pública, para favorecer un pluralismo posible" (Del Caz, Gigosos, Saravia, 2002: 102).

El conflicto se produce en la ciudad, en el encuentro de ciudadanos libres que son diferentes, el conflicto motiva la política y hace del espacio público (el espacio del encuentro) el espacio político donde se desarrolla el arte del diálogo, de la negociación y del consenso. El conflicto es inherente a la misma, igual que la convivencia. Conflicto y convivencia son las dos caras de la misma moneda. En la medida que el espacio urbano también es usado de forma diferenciada y en momentos también diferentes en función de las características específicas de las diversas redes, el espacio se encuentra en discusión permanente (HERNÁNDEZ AJA, 2003:) según el genero, la edad, la clase, la cultura... y todas esas condiciones tienen que proclamar una complementariedad y, a veces, simultaneidad en el mismo espacio público cruzándose y haciendo del espacio público un espacio complejo (variado, plural, de mezcla) y a la vez sencillo (percibido, 
controlado, legible, simbólico...) que precisa de una renegociación permanente de la complementariedad, de tal modo que nadie pueda quedar excluido o pueda sentirse perdedor. La ciudad es le lugar para el encuentro de las diversas identidades, lo que hace de la identidad algo que no puede construirse de manera exclusiva ni excluyente, el espacio público como espacio político debe orientarse a construir una identidad de identidades que englobe a todas, a la misma vez que defiende a cada una de ellas.

\section{La deconstrucción de la ciudad y del espacio público}

Llegados a este punto cabe preguntarse si la ciudad de la que venimos dando cuenta se corresponde con la ciudad que hoy experimentamos: el modelo metropolitano; y habría que preguntarse ¿son realmente ciudades, éstas grandes aglomeraciones urbanas? Una de las constataciones que podemos reseñar es que estas grandes ciudades son espacios crecientemente problematizados, en ellas se concentra cada vez más la pobreza y la población excluida, y se evidencia la desigualdad social como nunca antes, son el origen de la insostenibilidad ambiental y del cambio climático, en ellas se constatan la mayor tasa de suicidios y enfermedades mentales, en ellas la soledad y la anomia atormenta a millones de personas, y también en ellas los síntomas de violencia urbana son crecientes, aún así nos sigue fascinando la ciudad en el contexto de una sociedad de consumo de masas. ¿Es esto realmente la muerte de la ciudad?, anunciada por los sociólogos urbanos en la década de los 60, como Jane Jacobs o Henri Lefebvre.

Tradicionalmente la densidad, la variedad y la dimensión eran las variables que definían la física social de una ciudad, es decir, una cantidad de sujetos y actividades variadas y mezcladas en interacción sinérgica. Si bien el modelo urbano que surge y se consolida con la industrialización y la extrema mercantilización desborda esos atributos propios de la ciudad. Los ámbitos urbanos crecen desmesuradamente dejando atrás su escala humana, se desdensifican extendiéndose como una mancha de aceite por el territorio haciendo de las ciudades espacios inabarcables, difíciles de percibir en su totalidad, e insostenibles ambientalmente, distanciado a unos de otros. La ciudad deja de ser accesible y solo podrán abarcarla, transitarla y vivirla en su totalidad los que tienen recursos privados de movimiento. Simultáneamente, el modelo urbano moderno apuesta por la zonificación de las funciones urbanas separando espacios de residencia, de trabajo, de consumo,... y segregando también los crecientes atributos de desigualdad. La ciudad industrial, primero, y después la ciudad postindustrial, que separa espacios y grupos sociales no lo hace de forma inocente ya que busca, entre otras cosas la ocultación del conflicto y de los "conflictivos", el aislamiento, la separación, la segregación de los conflictivos es la forma de no reconocer la desigualdad, es la forma de ocultar el conflicto y con ello de acabar con la convivencia. No es ciudad, no es lugar, no hay convivencia, donde se produce la agorafobía (el miedo-rechazo al espacio público) y la xenofobia (el miedo-rechazo a los diferentes).

De acuerdo Jordi Borja las diferentes presiones sobre la ciudad han producido un triple proceso negativo: fragmentación (zoning urbano), disolución (difusión urbana) y 
privatización (extrema mercantilización de la ciudad) (Borja, 2003, 163). Veamos estos procesos detenidamente, ya que a su vez desatan procesos que amenazan seriamente al espacio público y los derechos de ciudadanía. En primer lugar, la zonificación o "zoning urbano" queda bendecido en la llamada "Biblia de los urbanistas" (Labesse, :1973), o Carta de Atenas (1942), que desde un intento del más puro dirigismo racionalista viene a proclamar la separación de las funciones urbanas ${ }^{1}$ en aras de la satisfacción de las necesidades humanas, ${ }^{2}$ puestas en entredicho por la insalubridad que resultaba de la promiscuidad entre las funciones urbanas y el hacinamiento característico de los efectos provocados por la revolución industrial sobre las ciudades. Sin embargo, los propios efectos perversos de la zonificación urbana se vienen expresando reiteradamente desde distintas perspectivas.

Con la consolidación de la sociedad industrial aparecen la planificación y los planificadores, pero también se produce una ruptura de la ciudad y de lo ciudadano. A medida que se produce el crecimiento del espacio urbano y con ello su funcionalidad, el seccionamiento espacial cobrará mayor importancia, pudiéndose caracterizar básicamente tres categorías espaciales segregadas: El espacio de la producción (del trabajo-empleo-asalariado), el espacio de la reproducción (doméstico) y el espacio de la distribución (gestión y consumo). La necesidad consiguiente de procurar la comunicación y la movilidad entre las diversas partes complejas de la metrópoli presupone la existencia de un cuarto tipo de espacio, éste más lineal y en forma de malla, que se refiere a todo lo relacionado con las infraestructuras de conexión entre fragmentos urbanos (infraestructuras del transporte y redes de comunicaciones entre los espacios separados). Las unidades urbanas especializadas, unifuncionales, son unidades parciales y por tanto simples, la vida cotidiana en una función parcializada es una cotidianeidad unidimensional, pero a la vez el sujeto «móvil» que distribuye su tiempo en vidas separadas y desplazamientos entre ellas en un vasto territorio urbanizado se convierte en un «yo» escindido y en una víctima de lo simple-complicado (contrapuesto a sencillo-complejo) que imprime el modo de vida metropolitano. Los vínculos sólidos, flexibles, accesibles, sencillos, son sustituidos por los vínculos líquidos, rígidos, movibles, complicados.

\footnotetext{
${ }^{1}$ En dos artículos de la Carta de Atenas se explicitó claramente este aspecto: Art. 77: «... Las claves del urbanismo radican en cuatro funciones: habitar, trabajar, recrearse (en las horas libres) y circular...»; Art. 78 «...los planes determinarán la estructura de cada uno de los sectores atribuidos a las cuatro funciones básicas y fijarán su respectivo emplazamiento en el conjunto urbano...». En ese mismo sentido Le Corbusier en sus «Principios de urbanismo» (1979) apuntilla, desarrollando los postulados de la Carta de Atenas: «La zonificación es la operación que se realiza sobre el plano urbano con el fin de asignar a cada función y a cada individuo su lugar adecuado» (TOBÍO, 1996: 62).

2 Para Le Corbusier -Sin duda considerado el más fiel representante del modernismo y de la defensa de la zonificación urbana- la satisfacción de las necesidades humanas debe alcanzarse a través de la función humana, lo que le lleva a proclamar las «necesidades únicas», que son necesidades-tipo comunes a toda condición humana, la «función-tipo», «la emoción-tipo»... (LE CORBUSIER, 1978) Le Corbusier, desde la perspectiva aquí adoptada, viene a confundir lo que son las necesidades humanas con los satisfactores de esas mismas necesidades.
} 
Se evidencia, junto al cambio cuantitativo, un cambio cualitativo. Emerge la ciudad del «fragmento» frente a la ciudad como «cúmulo de sedimentos»; siendo la variable tamaño crecientemente incontrolada. Es una ciudad ahistórica que, construida extensamente bajo un rápido y desordenado desarrollismo ${ }^{3}$ y a una escala que se escapa al control individual y colectivo, imprime una funcionalidad que viene determinada por el mercantilismo como hecho intrínseco. Se disocia la instancia ciudadana y junto a ella se enajena al ciudadano del hecho urbano, en palabras de René Schoonbrodt «el urbanismo funcionalista basado en la zonificación aísla los medios sociales ente sí y, en consecuencia, tanto la sociedad en su conjunto como los distintos medios sociales se hacen ajenos los unos a los otros» (Schoonbrodt, 1994: 393).

Precisamente es esto lo que lleva directamente a otras consecuencias; unas más sociales: se produce una «parcelización de la existencia humana» (Del Acebo, 1993: 164-165), una ruptura del tiempo en la vida cotidiana y una división del espacio según la condición social: edad, profesión, procedencia, étnica, religión, clase, género ${ }^{4}$...; otras más ambientales: ocupación de suelo y desintegración de enclaves naturales por la necesidad de la construcción de grandes infraestructuras que permitan la movilidad cotidiana entre los espacios separados y que suponen de facto la creación de barreras y fronteras en el organismo urbano (se enfrenta el concepto de movilidad, con el de accesibilidad. La existencia de grandes distancias y la creación de barreras infraestructurales suponen una pérdida en la calidad en la accesibilidad a determinadas funciones urbanas especializadas según que tipo de sectores sociales). Adaptación, por tanto, del territorio y de la ciudad al uso del vehículo motorizado, que se deriva de un aumento paulatino de las distancias entre los elementos urbanos funcionales, y que conlleva un despilfarro energético y un incremento de la congestión y de los niveles de contaminación.

En segundo lugar, la dispersión-difusión de la urbanización, aspecto que además es inseparable de la zonificación, viene a establecer una nueva paradoja en el proceso de urbanización de los países desarrollados. Por primera vez la expansión del hecho urbano se produce al margen de los comportamientos demográficos, es decir, mientras que la población de las grandes ciudades del mundo desarrollado se mantiene estable o incluso decrece ligeramente, la expansión del espacio construido alcanza cotas de ocupación de suelo inusitadas gracias al desarrollo de una tupida red de infraestructuras para el transporte. Esa dispersión de la urbanización no tiene umbrales territoriales fácilmente observables, ni está distribuida de forma biunívoca en el espacio físico (Martinotti,

\footnotetext{
${ }^{3}$ Según Ekhart Hahn el índice de ocupación urbana de la superficie se ha multiplicado por 10 en los últimos 100 años (HAHN, 1994).

4 A este respecto existe una amplia literatura basada en investigaciones empíricas desarrolladas desde la Escuela de Chicago y más recientemente desde la Sociología y la Antropología Urbana que muestran la segregación del espacio según la condición social diferenciada. Sobre la separación de funciones relacionadas con el genero, una de las perspectivas de estudio de mayor interés en la actualidad, cabe referenciar la constatación de como la zonificación implica también una división del espacio según el género (TOBÍO, 1996).
} 
1990), la contigüidad de espacios urbanos funcionales y a la vez la continuidad del espacio urbano por todo el territorio son la expresión de esa paradoja parcial del metropolitanismo. Si bien ese nuevo orden territorial único, que hace desaparecer la vieja dicotomía entre campo-ciudad para introducir nuevas contradicciones, significa que se pierde la idea de la ciudad entendida como «lugar» de acogida que era reconocible sobre la vasta extensión del «no-lugar» propia de una ciudad de los flujos en donde circulan los objetos-mercancía y los sujetos-mercancía (sujetos objetivados): sujetos usuarios, clientes, administrados, productores, consumidores, pero dudosamente ciudadanos.

De este modo, la movilidad y la velocidad es motivada en un modelo metropolitano que implanta un modo de vida fundamentado en el automóvil, que proclama un uso individualizado del espacio no-relacional, de la ciudad de los flujos. Sin embargo, este proceso se manifiesta cada vez más como un factor de exclusión social, ya que los colectivos segregados espacialmente tienen menos posibilidades de movilidad física para desplazarse de unas funciones a otras (por ejemplo de la residencia al trabajo) lo que conlleva una prolongación de la jornada laboral al sumar a esta el tiempo de dedicado a los largos desplazamientos y la dificultad para acceder a servicios urbanos básicos.

En tercer lugar, la extrema mercantilización de la ciudad en un contexto de insaciable neoliberalismo nos ha llevado a lo que se ha denominado como una "segunda generación" de privatizaciones que se corresponde con lo que David Harvey ha identificado como "Acumulación por desposesión" (Harvey, 2004) como actualización y continuación de la "Acumulación originaria de capital" consistente, tanto en el acoso y derribo a la propiedad pública, como de la propiedad comunitaria. Así la mirada mercantilista ha motivado la privatización de servicios públicos (transportes, mantenimiento urbano, equipamientos, gestión de residuos, gestión del agua, funerarias, etc.), la conversión del suelo y la vivienda en recurso especulativo, la mercantilización de recursos ambientales, la privatización de espacios públicos, la creación de seudo espacios públicos simulados, la mercantilización de las relaciones sociales de orden primario (cuidado de los hijos, de las personas dependientes), etc., todo ello ha proporcionado una "gestión mercantilista que acaba llevando la diferenciación de los usuarios por su capacidad de pago..." (Saravia et al., 2002: 33)

El impacto combinado de estos procesos que se refuerzan mutuamente ha contribuido a la destrucción del espacio público como espacio de ciudadanía (Borja, 2003: 163-164). Particularmente paradigmático de la citada combinación es la implantación de las grandes superficies comerciales, enclaves exclusivos para el consumo convertido en ocio, o viceversa, convenientemente separados de la residencia precisan del uso del automóvil destruyendo, además, el pequeño comercio de barrio y sustituyendo la accesibilidad por la movilidad. Asimismo el gran centro comercial es un sucedáneo del espacio público destinado al consumo como simulación del ocio y de la libertad individual, es causa y efecto, de la pérdida del espacio público. La gran 
superficie comercial, como espacio privado, se basa en el derecho de (no) admisión y no todos pueden acceder, el nivel de rentas es ya de por sí un sesgo significativo, pero en todo caso nadie puede sentirse parte de ese espacio, ni participar del mismo, ni disfrutar de la improvisación del encuentro con el conocido. Los sobreestímulos que irradia ese no-lugar oculta el significado del mismo. Si alguien mendiga en ese espacio, o juega con una pelota, o reparte octavillas, o hace mimo, o toca música... será inmediatamente expulsado.

De este modo, el triunfo del paradigma funcionalista no solo ha terminado desmembrando la ciudad, sino que acompañando ese proceso también ha segregado socialmente a las redes sociales. Una estructura social extremadamente fragmentada, como es la de las sociedades capitalistas avanzadas, tiene sus consecuencias sobre la disposición, dominio y uso de los espacios públicos. La creciente distancia social entre los distintos segmentos que conforman la estructura social conlleva separación y segregación espacial y este fenómeno a su vez implica la imposibilidad o al menos una dificultad significativa en el encuentro y en el intercambio de las experiencias vitales de diferentes redes sociales, haciendo de ellas estructuras informales endogámicas, crecientemente encerradas sobre si mismas, donde la confianza interna contrasta con la desconfianza con el exterior de la red. Cuanto mayor es la separación de las funciones urbanas, de los colectivos, de las actividades, más se debilita el capital social y las relaciones sociales de confianza.

La combinación, en las sociedades capitalistas, de la separación de las funciones urbanas con la separación de las culturas, de las clases sociales y de los fragmentos de clase, esta incidiendo en la destrucción del espacio público, despojándole de sus funciones tradicionales de encuentro, entre ellas su función política del compartir, del negociar, del consensuar, del manejo del conflicto que es controlable gracias al contacto cotidiano entre sujetos y colectivos deferentes. La distancia entre los grupos se acrecienta en sus distintas perspectivas: social, económica, cultural, ambiental... incorporando nuevas e inquietantes perspectivas del conflicto urbano en forma de violencia urbana y particularmente de enfrentamiento entre redes y fracciones de clase. La urbanización funcionalista, en su doble separación física y social, de espacios monocultivo y de grupos endogámicos, impide de facto la producción social del espacio, la construcción conjunta de la interpretación y la democratización del mismo. La imposición de las clases y grupos dominantes en la producción de formas espaciales hace de esta producción un factor de dominación y de explotación (Castells, 1979) que con la creciente fragmentación de la estructura social conlleva el paso de una antagonismo relativamente simétrico (existencia de procedimientos de negociación y pacto entre clases en la sociedad urbana industrial) a una dinámica segregadora que "conducen -en palabras de Jordi Borja- a formas conflictivas, dispersas y asimétricas, difíciles de encuadrar en procesos negociadores, que pueden derivar en expresiones de violencia anómica" (Borja, 2003: 49). Ello se produce como consecuencia de la presencia creciente de sectores excluidos a los que no se les reconoce plenamente los 
derechos de ciudadanía. Los conflictos derivan entonces al enfrentamiento entre segmentos de las clases subalternas y las denominadas infraclases, en vez de entre los grupos desfavorecidos y el poder instituido, la solidaridad propia de las clases subalternas en la ciudad industrial deja paso a la subdivisión de clases por el grupo étnico que tiende a impedir la solidaridad (Colm Hogard, 2002: 91).

La segregación social, la distancia física, social y económica de las redes, clases, culturas, genera desconfianza y miedo al otro. Emergen las comunidades cerradas (gated communities), los barrios gueto y los barrios bunker (Naredo Molero, 1998), los condominios exclusivos que se construyen como "espacios defendibles" y levantan barreras a la democracia destruyendo la función del espacio público y el derecho a circular, a estar en determinados lugares, a encontrarse. Una sociedad que se construye sobre comunidades cerradas, que genera barreras y terrenos de nadie es propensa, en consecuencia, al descuido urbano y a la violencia urbana. El círculo vicioso de la violencia urbana produce la agorafobia, el miedo al espacio público, y éste abandonado se hace más vulnerable, de dominio y de uso menos complejo. La pérdida de variedad en su dominio y en su uso le debilita también como espacio político, ya que la política no puede ser bajo el miedo, no puede ser sin el encuentro, sin el diálogo, sin la comunicación. La agorafobia, va acompañada de la aporafobia y de la xenofobia, el miedo a la pobreza y a los diferentes hace que el espacio público sea abandonado por determinados segmentos de la estructura social y dejado exclusivamente a otros, construyendo sus propias fortalezas que les protegen de los indios metropolitanos que pugnan por sobrevivir en un medio urbanizado (que no urbano) cada vez más hostil e ingobernable.

\section{(Re)volver a la ciudad, reconstruir el espacio público, reconquistar el espacio político: redescubrir la ciudadanía}

Es incuestionable que nuestro modelo metropolitano, que nuestro modo de vida precisa de una reorientación que se hace cada vez más urgente. La nueva conciencia que sobre ello se esta generando no es desdeñable y tiene su traslación en nuevas miradas sobre el individuo y la ciudad, y el vínculo que sobre ellos se establece. Son cada vez más las reflexiones, las iniciativas, las experiencias innovadoras que nos indican el desarrollo de nuevas estrategias que caminan y proclaman una democracia participativa en la gestión de los recursos y del territorio, una nueva ciudadanía que se renueva con la incorporación de nuevos derechos, sobre todo, culturales y ambientales. Su dispersión, y su aún escasa articulación hace poco visualizable su desarrollo, pero los síntomas aparecen a una velocidad vertiginosa en múltiples formas asociativas, en nuevos movimientos sociales, en nuevas formas de gobernar, en experiencias innovadoras participativas, en buenas prácticas urbanas... Esta amalgama compleja de factores la podemos desarrollar en sus dimensiones culturales, sociales, políticas, de naturaleza económica y de carácter físico-ambiental, las cuales también las podemos considerar como condiciones necesarias para reconstruir el espacio público como espacio político. 
En primer lugar, la interculturalidad alcanzada en un mundo globalizado instituye nuevos vínculos entre el sujeto personalizado y lo universal, haciendo de éste un ciudadano a la vez local y universal que adquiere una nueva ética. Tal como hemos expresado en otro trabajo (Alguacil, 2007: 40) emerge una nueva cosmología mundialista, una nueva conciencia, un nuevo sujeto protagónico, es el regreso del sujeto que diría Jesús Ibáñez (1991), que se rearma en la ética a través de renovados valores morales frente al pensamiento técnico, científico, mercantilizado. Haciendo nuestras las palabras de Alain Touraine en uno de sus últimos libros "vemos también como el juicio moral recupera terreno frente al pensamiento técnico y científico. El movimiento ecologista nos ha enseñado a reconocer nuestros deberes respecto de la naturaleza, lo que no nos ha llevado a fundir la cultura en la naturaleza, sino, al contrario, a hacer penetrar el juicio moral en el dominio de la naturaleza" (Touraine, 2005: 177), lo que es tanto como reconocer que los sujetos ganan capacidad de pensamiento y de acción, obtienen autonomía desde la dependencia ecosistémica, de tal modo que el sujeto tiene conciencia de sí en la medida que forma parte activa de la naturaleza y de la sociedad. Es decir, lo personal es político, "es afrontar -en palabras de David Harvey- la cuestión de la persona y del cuerpo como el momento irreducible (definido en una determinada escala espacio-temporal) para basar toda la política y la acción social. Pero el individuo, el cuerpo, el yo, la persona (o cualquier otro término que deseemos utilizar) son un fluido constructo social más que una entidad absoluta e inmutable fijada en cemento" (Harvey, 2003: 270).

Precisamente, el conocimiento sobre los límites ecológicos y la preocupación por las futuras condiciones de vida en el planeta (conciencia) vuelven a poner en escena renovados aspectos de la subjetividad humana y de la autonomía ética. Surge así una nueva paradoja: la autonomía individual se obtiene gracias a la pertenencia a un contexto relacional, o a un ecosistema, y ese pertenecer a un entorno significa un cierto grado de dependencia. La autonomía individual no puede pensarse sin la autonomía de los otros, o sí se prefiere, de la dependencia de los otros. La autonomía desde una perspectiva ética representa, pues, la complementación de la identidad y de la alteridad. La comunicación relacional implica la relación simétrica entre emisor-receptor y receptor-emisor que interaccionan sobre la base de una identidad común (los signos y señales de sus comunicaciones no sólo encauzan información, sino también identificación) y que supone el reconocimiento del otro (alteridad) y de sí mismo a través de ese reconocimiento. Dice Touraine "que se siente sujeto solamente aquel o aquella que se siente responsable de la humanidad de otro ser humano. Es reconociendo los derechos humanos del otro como me reconozco a mí mismo como ser humano..." (Touraine, 2005: 169), es reconociendo los derechos y las necesidades humanas ampliados donde regresa el sujeto en esa tensión dialéctica que se produce entre lo local y lo global, el individuo y el colectivo, la singular y lo universal.

Así, el extremo individualismo desarrollado en la metrópoli, vinculado a la mercantilización, "también puede propiciar el desarrollo de una conciencia ampliada 
capaz de configurar nuevos espacios propicios al ejercicio de las virtudes cívicas...., cuando el yo actúa, en nombre propio y asumiendo la responsabilidad de sus actos, adentrándose en lo universal y relegando su egoísmo" (Barry, 1999: 145). Ésta nueva ética se obtiene en las redes interactivas donde se adquiere una «capacidad estimativa» 5 que orienta la acción humana buscando el equilibrio entre la libertad individual y la vinculación colectiva, entre lo micro-social y lo macro-social, entre las emociones y la(s) razón(es). Esa capacidad estimativa es reforzada por la comunicación y el conocimiento, que a su vez estimulan el devenir consciente. Y esta ética discursiva proyectada en acción colectiva es la que nos lleva a la confirmación de como es a través de los nuevos movimientos sociales, que buscan no tanto la reivindicación de los derechos, de la dignidad, de los nuevos valores, como de hacerlos realidad y de aplicarlos directamente cuando tienen oportunidades, la que permite la construcción de un sujeto-persona que recupera un sentido de la vida ético que esta dando pie a nuevas prácticas urbanas participativas.

Desde una segunda perspectiva, más en el ámbito de lo societario, se puede argumentar como es en la ciudad donde el individuo tiene acceso a múltiples puntos de vista que se mezclan y que se concretan en una compleja constelación de redes y organizaciones. No olvidemos que es en la ciudad donde surgen la organización y la asociación que con la creciente complejidad y fragmentación lleva a ésta constelación de estructuras de acción política a buscar su articulación (buena muestra de ellos es el denominado movimiento antiglobalización). Pero es precisamente en el ámbito de la ciudad, en el ámbito local, en un contexto de proximidad, de contacto directo, de confianza, de conocimiento mutuo, donde los sujetos pueden entrar en estrategias de construcción conjunta que les permita generar y acceder a estructuras comunes de acción política. En el mundo local se encuentran organizaciones de orden gubernamental (gobiernos locales) y de orden societario (organizaciones y movimientos sociales). Ambos tipos de estructuras, por su posición privilegiada de proximidad y de ser potencialmente difusores de los principios universalistas son escuelas de democracia.

Es a través de éstas estructuras donde se puede acceder a las habilidades para la participación política $\mathrm{y}$, por tanto, a adquirir la condición de ciudadano pro-activo. Estas estructuras se encuentran en mejor disposición para incorporar a los ciudadanos a procesos de comunicación y de deliberación y, en consecuencia, están en mejor disposición de transmitir a los ciudadanos las destrezas necesarias para desenvolverse en la esfera de la política. Se trata, en definitiva, de un proceso recurrente dónde la comunicación, el conocimiento y la reflexividad sobre la acción permiten la adquisición de la (co)responsabilidad social y llevan a la emergencia de la nueva

\footnotetext{
${ }^{5}$ Vamos a entender la «capacidad estimativa» del sujeto de una forma particular, y siempre dentro del sentido dado a la «autonomía crítica»: Como el nivel de conciencia adquirido a través del acceso al conocimiento (de continua adaptación al medio, a los recursos y a las condiciones) y a los procesos de comunicación fluida (de reciprocidad en la relación con otros sujetos).
} 
conciencia necesaria para desarrollar las nuevas dimensiones de ciudadanía. De tal modo, que la incorporación de los ciudadanos a la globalización (como un continuo de esferas de soberanía y diversidad cultural complementarias y potencialmente articuladas) solo puede optimizarse a través de las redes y movimientos sociales de arraigo territorial y de las estructuras gubernativas descentralizadas, aunque precisan de procedimientos orientados a la articulación entre sí y la conexión con otras estructuras descentralizadas y globales. En consecuencia, la emergencia de estos nuevos derechos de ciudadanía vienen asociados al surgimiento de renovados actores (gobiernos locales, movimientos sociales, tercer sector) que ponen de relieve nuevas subjetividades en la conquista de una ciudadanía que explora nuevas dimensiones de la misma.

Esta perspectiva, nos lleva al espacio de la política donde se puedan desarrollar aquellas estrategias políticas que generen nuevas oportunidades para la implicación de los ciudadanos en la gestión de la ciudad y de los espacios públicos. Para que ello sea posible la participación tiene que recuperarse plenamente como necesidad humana que en el actual modelo social mercantilizado se encuentra constreñida y oculta, al fundamentarse este paradigma en estrategias inhabilitantes para con los sujetos que son sustituidos por especialistas y profesionales de la política.

Esa recuperación precisa, en consecuencia, de innovaciones capaces de traducir la participación en comunicación relacional para la acción ciudadana conjunta (PUIG, 2003) donde el papel de los profesionales de la política sea el de implementar los procedimientos para que los ciudadanos tengan capacidad plena para participar. El sentido relacional de la participación tiene varias miradas interpenetradas que permiten acoplar la participación a la propuesta de democracia participativa como una síntesis de la alianza cada vez más necesaria entre las estructuras gubernamentales y las entidades y movimientos sociales.

En primer lugar, es necesaria una nueva cultura política fundamentada en la habilitación de los ciudadanos para que puedan acceder a la información y el análisis de sus condiciones de vida y obtengan capacidad de decisión en aquellos aspectos que les afectan directamente, en el acceso, en definitiva, a la satisfacción de las necesidades humanas desarrollando satisfactores de carácter sinérgico donde la transversalidad de la participación aparece como procedimiento inalienable. Se trata de reconocer el estatus pleno de acción política de los ciudadanos considerando el desarrollo de su autonomía crítica. La formación para la ciudadanía será un elemento fundamental para ello y la promoción de las organizaciones sociales de base, como escuelas de democracia, el escalón, junto con unos gobiernos locales con recursos suficientes, serán dos aspectos cruciales para que ello sea posible, a lo que habría que añadir la necesidad de un sistema educativo adaptado a tales requerimientos.

En segundo lugar, una nueva cultura política, precisa desarrollar procedimientos que combinen adecuadamente los principios de solidaridad interterritorial y la 
subsidiariedad, según el cual todo aquello que pueda ser autodeterminado o autogestionado con eficacia y responsabilidad a un determinado nivel ciudadano (inferior o de escala más reducida) no debe determinarse o gestionarse en un nivel superior o de escala mayor. Dicho de otro modo, que la satisfacción de las necesidades y demandas debe resolverse en el nivel más próximo a los ciudadanos que sea posible. Es necesaria, desde esta perspectiva, una nueva cultura política encaminada a la construcción un Estado relacional descentralizado, articulado y participativo que produzca nuevas oportunidades para la ciudadanía promoviendo organizaciones en red y una nueva legitimidad de un Estado democrático reconstituido desde su base, desde las ciudades, desde los municipios.

Para el desarrollo de esta perspectiva relacional de la administración proponemos considerar como elemento clave la combinación entre el principio de descentralización y de participación, lo que debe abordarse diferenciando entre distintos aspectos que completan la idea de descentralización y que podemos identificar con tres des: ${ }^{6}$ la desconcentración político-administrativa, la descentralización política y la desburocratización en lo sociopolítico. Representa tres ejes de horizontalidad: Un eje económico-administrativo definido por la distribución de los recursos de la economía pública, un eje político definido por la transferencia de competencias, y un eje sociopolítico identificado con la distribución del poder. Estos tres modos son interdependientes, se transpenetran entre sí y su distinción establece distintas perspectivas para significar aspectos ineludibles para la democratización de la sociedad (combinación de la democracia deliberativa, participativa y asociativa), lo que no debe confundirse con lo que podríamos identificar como la "desestatización" que entiende la descentralización en un eje vertical de tal modo que las decisiones tienden a centralizarse mientras la ejecución de las mismas viene a desconcentrarse por delegación impositiva y por privatización de los servicios públicos. Esta mirada de la descentralización apuesta por la democratización interna de la administración pública como manera ineludible para incrementar la calidad de la democracia, poniendo de relieve la necesaria articulación entre la democratización de la política y la democratización de la administración. Para que esto sea posible se precisa de dos condiciones: en primer lugar, la participación debe desplazarse "de la política a las políticas” y, en segundo lugar, “de la universalidad a la red” (Brugué, Gallego, 2001).

Finalmente, cabe estimar los instrumentos de los que se puede dotar esta perspectiva relacional de la política para desarrollar las estrategias de incremento de la calidad de la democracia y de la participación. Nos estamos refiriendo a las múltiples innovaciones de carácter democrático que se desarrollan en los últimos años, sobre todo, en el ámbito municipal y que son buena muestra del camino a seguir para reconquistar el espacio público. Estos son muy variados pudiendo distinguir entre innovaciones de

\footnotetext{
${ }^{6}$ Se realiza un desarrollo más profuso de esta perspectiva en Alguacil, J. (2003): Equipamientos municipales de proximidad. Plan estratégico y de participación. Gijón, Trea / Kaleidos.red.
} 
base asociativa (cuando los procedimientos sólo posibilita el acceso a los ciudadanos organizados), de base personal (cuando los procesos participativos permiten el acceso a los ciudadanos), de base mixta (cuando los procedimientos incluyen de forma simultánea y compatible las dos anteriores) (Font, Gomá, 2001). Así los Presupuestos Participativos, La gestión compartida de equipamientos, los Jurados Ciudadanos, Los Grupos Temáticos, las Encuestas Deliberativas, las Asambleas Ciudadanas, los Fórums Temáticos, La Investigación-acción participativa, los Planes de Desarrollo Comunitarios, etc. son todos ellos instrumentos que se han ido incorporando en muchas ciudades en los últimos años permitiendo deliberar y decidir a los ciudadanos sobre los asuntos públicos y la gestión de la ciudad.

Una cuarta dimensión de la que hay que dejar constancia es la perspectiva económica que en gran medida deriva de la estrategia de desconcentración políticoadministrativa como eje económico-administrativo y que pone de relieve la existencia de una economía pública que interactúa fuertemente con otros tipos de economía. En este sentido, considerando la existencia de una economía de la diversidad: economía pública, economía de mercado, economía popular y una economía social (Coraggio, 1999), el desequilibrio vendría establecido por la dominancia de la economía de mercado cada vez más frecuentemente promovida y apoyada desde la economía pública, por ejemplo, a través de las privatizaciones. Cabe preguntarse sobre la ética que le corresponde a la economía pública sustentada por el conjunto de la sociedad. Si consideramos como uno de los objetivos de una administración relacional el incrementar la calidad de la democracia y la participación ciudadana, ésta no debe establecer crecientes compromisos con la lógica del lucro constituida por el beneficio privado y unas estructuras nada o poco democráticas representadas por las empresas capitalistas tradicionales. Por el contrario, la reconstrucción del espacio público como espacio político tiene su prolongación en el ámbito económico y desde la economía pública debe favorecerse el apoyo a una economía democrática como la que pueda representar la economía social y a una economía de proximidad representada por la denominada economía popular (por ejemplo el pequeño comercio o las microempresas), ambas están más integradas en el territorio, tienen mayor capacidad de creación de empleo y de satisfacer necesidades locales, y son susceptibles de mayor responsabilidad social, política y ambiental.

En consecuencia, como ya hemos expresado en otro trabajo (Alguacil, 2006: 34) la derivación de la gestión de los servicios y prestaciones públicas hacía fuera de las estructuras administrativas solo se debería hacer desde la convicción y la aplicación operativa de los principios de cooperación, corresponsabilidad, universalidad y participación, y de los valores éticos que de estos principios se derivan. Cabe pensar, entonces, en una orientación de las tan traídas y llevadas externalizaciones como una estrategia que facilite la implantación de políticas equitativas, que fortalezca los derechos ciudadanos y que favorezca un desarrollo humano sostenible, dimensiones todas ellas que se desarrollan de forma interpenetrada en el campo de todo lo que 
podríamos considerar como sector público no-estatal (ciudadanos activos, tercer sector, economía social).

Una última mirada, referida esta vez a los aspectos de naturaleza física, de estructura urbana, que no se pueden imaginar sino es combinación con las dimensiones anteriores es la que se refiere a la escala urbana necesaria para reconstruir el espacio público y el espacio político. Recuperar la ciudad requiere la reconstrucción de unidades urbanas con identidad propia, descentralizadas y complejas internamente, con una autonomía política significativa, densidad, variedad y una escala humana que facilite su percepción y apropiación. Cualquiera tiene derecho en su barrio a tener empleo, equipamientos, entidades sociales, espacios públicos, elementos monumentales, elementos de centralidad, de singularidad, etc. como cualquier ciudad. Es necesario, por tanto, descomponer-recomponer las grandes conurbaciones en múltiples ciudades integradas internamente e interconectadas externamente entre sí, haciendo compatibles la ciudad de "los lugares" (de la accesibilidad) con la ciudad de "los flujos" (de la movilidad).

La ciudad a escala humana significa recrear la máxima complejidad accesible, es decir, lo suficientemente grande para el anonimato y la variedad de relaciones, pero a la vez lo suficientemente pequeña como para mantener una red social densa (en el espacio), intensa (significativa) y continua (perdurable y sostenible en el tiempo), en contraposición al efecto metropolitano donde se produce lo contrario, o se tiene exceso de estímulos relacionales en un territorio extenso que hace que los vínculos sean más débiles y las relaciones más esporádicas y efímeras o, por el contrario, se sufre el aislamiento y la soledad. La libertad individual y la identidad colectiva no deben ser inconciliables, la libertad de elección precisa de variedad de opciones compatibles y sinérgicas. Para que esto sea posible es imprescindible eliminar las barreras y reconstruir fronteras simbólicas y porosas que permitan la continuidad y la diferenciación de espacios sin que nadie pueda sentirse segregado o descolocado en cualquier barrio de la ciudad, y todos puedan percibir cual es su "lugar", cual se su barrio.

El barrio es fundamental para reconstruir la ciudad, la revalorización y reconocimiento del barrio en la teoría urbanística viene a ser considerada como una escala adecuada para resolver los múltiples, graves y crecientes problemas de las grandes ciudades. Al respecto, la Agenda Hábitat, en su afán de promover la ciudadanía expresa que "el barrio es una escala fundamental para el análisis de los problemas económicos sociales, urbanos o ambientales de las ciudades, que pone en contacto las políticas con la realidad social, y facilita la definición de soluciones y la instrumentación en forma interactiva con los agentes sociales locales". 7 Como muy bien expresa Pedro G. Buraglia: "El barrio opera como el eslabón de una cadena entre el ser

\footnotetext{
${ }^{7}$ Agenda Habitat / Habitat II. Primer documento de trabajo. Agenda Hábitat España contribución de las ciudades al desarrollo sostenible. Conferencia de Naciones Unidas sobre asentamientos humanos. Estambul, 1996. MOPTMA, Dirección General de Actuaciones Concertadas en las Ciudades (Feb. 1996). (Pág. 71-72).
} 
individual y el ser social, facilita la convivencia solidaria, la interacción entre grupos y personas, es también el espacio para el aprendizaje y formación del ser social, que opera como el lugar para iniciarse en una determinada actividad trascendiendo el marco puramente familiar, por ejemplo, las primeras relaciones amorosas, los primeros contactos deportivos o sociales o las primeras manifestaciones políticas" (Buraglia, 2005).

Si bien, para reorientar el modelo metropolitano, los barrios tienen que ser equiparables a una ciudad, con toda la variedad y con todos los recursos propios de ésta. El modelo urbano teórico es una propuesta que podemos concretar tal y como se conceptualiza en el libro "La ciudad de los ciudadanos" (Hernández Aja, Alguacil et al., 1997): Barrio-ciudad: lo consideramos como el primer escalón urbano con capacidad de sostener la complejidad y variedad propia de la ciudad histórica, permite albergar diversos estratos y distintas formas de vida y culturas. Es decir, es el modelo urbano que puede acoger la máxima complejidad asequible y permite el acceso a lo heterogéneo y a la responsabilidad social, teniendo capacidad para generar recursos propios. Suele coincidir con el ámbito de actuación de las asociaciones y debe de contener las dotaciones necesarias para el desarrollo de sus poblaciones, incluido algún equipamiento de rango ciudad que suponga un foco de atracción e identidad para el conjunto de los ciudadanos. La disposición de los espacios públicos, arracimados unos a otros, con sendas que les hacen accesibles, es lo que hace posible la vertebración de un Barrio-ciudad, es lo que hace posible la ciudad misma, como entidad y con identidad propia. El entramado de los espacios públicos, sean abiertos o edificios, deben estar asociados entre sí de modo que el sentido que obtiene cada espacio público viene dado por la relación con los demás, y deben estar concebidos y dispuestos de tal modo que no sea necesario ir a ellos exclusivamente, sino que te los encuentras en los trayectos cotidianos de la vida de barrio. Con un tamaño de población (no más de 50.000 habitantes y no menos de 20.000), el Barrio-ciudad obtendría capacidad de sostener la variedad de las diversas estructuras solapadas (demográficas, sociales, inmobiliarias, de actividades económicas, etc.) y abarcarse peatonalmente, en él el ciudadano es capaz de generar sentimientos de identidad y arraigo, de controlar el territorio, de acceder a las habilidades políticas y sociales; y se puede, así, identificar con su territorio estableciendo un equilibrio entre máxima libertad individual sin comprometer por ello la responsabilidad colectiva.

En definitiva, repolitizar la ciudad, recobrar la convivencia, precisa de la reconquista del espacio público como espacio relacional y polivalente, como espacio para la expresión y creatividad ciudadana, como espacio con capacidad para reordenar la conectividad y la accesibilidad entre las funciones urbanas, y como espacio con capacidad para motivar el acceso a la movilización y a la participación de los ciudadanos en los asuntos públicos. Una ciudad segura es una ciudad cuyos espacios públicos son reocupados, son transitados, son compartidos. El espacio público es, en definitiva, un ámbito privilegiado que ofrece oportunidades inestimables para estimular 
ESPACIO PÜBLICO Y ESPACIO POLIIIICO. LA CIUDAD COMO EL LUGAR PARA LAS ESTRATEGIAS DE

PARTICIPACIÒN Págs. 199-223

( ) EDITORIAL DE LA UNIVERSIDAD BOLIVARIANA DE CHILE, 2008

la participación ciudadana, lo que ayuda a prevenir y oponerse a las dinámicas excluyentes de la desmesurada mercantilización de las metrópolis posindustriales. 


\section{Bibliografía}

Acebo Del, E. (1993), Sociología de la ciudad occidental -un análisis histórico del arraigo. Claridad, Buenos Aires.

Agenda Hábitat España (2006), Contribución de las ciudades al desarrollo sostenible, Conferencia de Naciones Unidas sobre asentamientos humanos. Hábitat II, Primer documento de trabajo, Agenda Hábitat España, Estambul, MOPTMA, Madrid.

Algualcil, J. (2007), "Nuevos movimientos sociales: nuevas perspectivas, nuevas experiencias, nuevos desafíos", en Elizalde, A.; Osorio, J. y Weinstein, L. (Editores), El Azul del Arcoíris, Universidad de Bolivariana, Santiago de Chile, pp. 27-68.

Idem (2006), "Los desafíos del nuevo poder local: ¿Hacia una estrategia relacional y participativa en el gobierno de la ciudad?" en Alguacil, J. (ed.). Poder local y participación democrática, El Viejo Topo, Barcelona, pp. 9-47.

Idem (2003), Equipamientos municipales de proximidad. Plan estratégico y de participación, Trea / Kaleidos red, Gijón.

Alguacil, J. y Montanes, M. (1998), "La participación ciudadana en la transformación del paisaje urbano", en Actas de las IX jornadas sobre el paisaje, Asociación para el Estudio del Paisaje / CNEA, pp.139-156.

Auge, M. (1993), Los no lugares: espacios del anonimato. Una antropología de la sobremodernidad, Gedisa, Barcelona.

Barry Clarque, P.(1999), Ser ciudadano, Sequitur, Madrid.

Borja, J. (2003), La ciudad conquistada, Alianza Editorial, Madrid.

Brugue, Q.; Gallego, R. (2001), “¿Una administración pública democrática?” en Font, J. (coordinador), Ciudadanos y decisiones públicas, Ariel, Barcelona, pp. 43-58.

Buraglia, P. G. El barrio, desde una perspectiva socio-espacial. Hacia una redefinición del concepto. www.barriotaller.org [consulta: 08-05-2005]

Castells, M. (1979), La cuestión urbana, Siglo XXI, Madrid.

Caz Del, R.; Gigosos, P.; Saravia, M. (2002), La ciudad y los derechos humanos, Talasa, Madrid.

Colm Hogart, P. (2002), "Conformismo, libertad y planeamiento", en Informe de Valladolid: Los derechos humanos en la ciudad, Universidad de Valladolid, pp. 91-96.

Coraggio, J. L. (1999), "La economía popular es más que la suma de microproyectos (alternativas para el desarrollo humano globalizado)" en Política y Sociedad $N^{\circ} 31$, Universidad Complutense de Madrid, pp. 47- 
68.

Delgado, M. (1999), El animal público, Anagrama, Barcelona.

Doyal, L. Gough, I. (1994), Teoría de las necesidades humanas, Icaria/FUHEM, Madrid.

Max-Neff, M.; Elizalde, A. y Hopenhayn, M. Desarrollo a escala humana: una opción para el futuro, Development Dialogue, número especial, CEPAUR y Fundación Dag Hammarskjöld, Uppsala.

Font, J., Gomá, R. (2001), "La democracia local: un mapa de experiencias participativas" en Font, J. (coordinador), Ciudadanos y decisiones públicas, Ariel, Barcelona, pp. 61-76.

Hahn, E. (1994), "La reestructuración urbana ecológica”, en Estudios Territoriales-Ciudad y Territorio No 100-101, pp. 369-388.

Harvey, D. (2003), Espacios de esperanza, Akal, Madrid.

Idem (2004), El nuevo imperialismo, Akal, Madrid.

Held, D. (1997), La democracia y el orden global. Del Estado moderno al gobierno cosmopolita, Paidós, Barcelona.

Hernández Aja, A.; Alguacil, J.; Medina, M.; Moreno, C. (1997), La ciudad de los ciudadanos, Ministerio de Fomento, Madrid.

Hernández Aja, A. (2003), "Ciudadanía y espacio público: participación o segregación” en ALGUACIL, J. (ed.) Ciudadanía, ciudadanos y democracia participativa, Fundación César Manrique, Teguise, Lanzarote, pp. 15-39.

Idem (2000), "Barrios y equipamiento públicos, esencia del proyecto democrático de la ciudad»”,en Documentación Social de Cáritas Española, № 119, pp. 79-93.

Ibañez, J. (1991), El regreso del sujeto. La investigación social de segundo orden, Amerinda Estudios, Santiago de Chile.

Labasse, J. (1973), La organización del espacio. Elementos de Geografía Aplicada, Instituto de Estudios de Administración Local, Madrid.

Le Corbusier, Ch. (1979), Principios de urbanismo, Ariel, Barcelona.

Martinotti, G. (1990), "La población en la nueva morfología social metropolitana. Reflexiones a partir del caso italiano", en BORJA, J. ET AL. Las grandes ciudades en la década de los 90, Sistema, Madrid, pp. 65139.

Maslow, A. H. (1975), Motivación y Personalidad, Sagitario, Barcelona.

Naredo Molero, M. (1998), “Seguridad y ciudadanía: necesidad de un “pacto de convivencia”, [En línea] 
Boletín $C F+S, N^{o} 8, \mathrm{Html}$ http://habitat.aq.upm.es/boletin/n8/amnar. html [consulta: 22-07-2005]

Puig, T. (2003), La comunicación municipal cómplice con los ciudadanos, Paidós, Barcelona.

Tobio, C. (1996), “Zonificación y diferencias de género”, en Astrágalo $N^{o}$ 5. Espacio y genero: Itinerarios al paraíso, pp. 61-75.

Schoonbrodt, R. (1994), "La ciudad es la organización física de la coexistencia" en Estudios TerritorialesCiudad y Territorio N$^{\circ}$ 100-101, pp. 389-396.

Touraine, A. (2005), Un nuevo paradigma. Para comprender el mundo de hoy, Paidós, Madrid. 